\title{
The direction of time and Boltzmann's time hypothesis
}

\author{
A.Y. Klimenko \\ The University of Queensland, SoMME, \\ Brisbane Qld 4072 Australia, \\ email: klimenko@mech.uq.edu.au
}

Preprint: March, 2018

(published: Phys. Scr. 94, 2019, 034002)

\begin{abstract}
This work explores Boltzmann's time hypothesis, which associates the perceived direction of time flow with the second law of thermodynamics. We discuss mechanisms that can be responsible for the action of the second law, for directional properties of time and, ultimately, for the perception that past events cause future events. Special attention is paid to possibility of testing these mechanisms in experiments. It is argued that CP-violations known in particle physics may offer such an opportunity. A time-symmetric version of Everett's many-worlds interpretation (which is based on a thermodynamic interpretation of quantum collapses) is given in the Appendix.
\end{abstract}

\section{Introduction}

This work is dedicated to promoting and expanding Boltzmann's hypothesis linking the perceived direction of time to the second law of thermodynamics [1]. Ideas of Ludwig Boltzmann created many disputes and controversies during his life, but, nevertheless, left us a rich inheritance that forms the cornerstone of modern science. Objectively, many statements of Boltzmann lacked rigor and this, in combination with the traditional disbelief of old science in reality of molecules, invited now century-old criticism of his work. At the same time, Boltzmann's ideas displayed deep intuition that was fully understood and appreciated by his followers (who include such figures as Planck and Einstein) only after his death. Boltzmann's time hypothesis is one of these great ideas, whose importance could not possibly be understood, let along appreciated, during his times. Yet, the world is slowly moving towards the understanding of the perceived direction of time offered by Boltzmann more than a century ago.

There is a great number of publications discussing the direction of time from the perspectives of philosophy and physics. The topic is virtually infinite and the reader can be referred to the principal publications: books [2, 3, 4, 5] on the philosophical side and books [6, 7, 8, 9] on the physical side of the argument about the direction of time. In this respect, the present work offers only a brief introductory narrative explaining some preliminaries. The focus of this work is Boltzmann's view on the perceived direction of time, its implications and, most importantly, the possibility of experimental testing of the time mechanism, which is opened by the Boltzmann hypothesis. We pay attention to the Diosi-Penrose theory 7 suggesting that thermodynamic time can be induced by gravity, but note that this theory is just one of many possibilities. In the context of the Boltzmann time hypothesis, the microscopic effect of "flowing" time can be detected without assuming any specific time-generating mechanism, although this requires rare cases in particle physics known as $\mathrm{CP}$ violations [10. A number of terminological and methodological issues are discussed in the Appendix.

\section{Intuitive perception of time}

Perception of time is an inseparable part of our consciousness. Humans recognise their existence as individuals in the context of their awareness of time. This recognition, which we may call self-awareness, is focused on the present moment of time - the moment through which the past moments known to us 
are continuously appended by events from as-yet unknown future. Our intuitive perception of time is perhaps best described by the lines

That river of time keeps on flowing

The present turns into the past

taken from poem "The river of time" by Linda Ori. These poetic words give an accurate metaphoric account of our intuitive perception of time. This perception can be expanded into the following six principles of Hans Reichenbach [3]:

1. Time goes from past to the future

2. The present which divides the past from the future is now

3. The past never comes back

4. We cannot change the past but we can change the future

5. We can have records of the past, but not of the future

6. The past is determined, the future is undetermined

These principles would be considered by most people as being obvious, which reflects the strength of our intuitive perception of time. Yet, it is arguable that the perceived flow of time is only a metaphoric description of the reality encompassing groups of dependent events that follow each other in time. Zwart [11] analysed this problem and remarked:

There is no flow of time beside or beneath the flow of events, but the flow of time is nothing but the flow of events.

Hence, the flow of time is represented by chains of ordered events, while each of these events is associated with a certain time moment and creates conditions for the following event(s) to appear. The directions along this chain - the past and the future - are obviously not equivalent: we think that the future events depend on past events but not vice versa. This non-equivalence of two temporal directions (toward the past and toward the future) is called the arrow of time. In the context of this non-equivalence, we often say that the preceding events cause the following events. This leads us to concept of causality, which we understand very well at the intuitive level and expect that, with the use of causality, a more rigorous and justified account can be provided for our intuitive time experience. This view was articulated by Reichenbach [3] more than half-a-century ago:

Casual connection is a relation between physical events and can be formulated in objective terms. If we define time order in terms of casual connection, we have shown which specific features of physical reality are reflected in the structure of time, and we given an explication to the vague concept of time order.

This program of expressing our basic intuitive perception of time in terms of most fundamental casual relationship between events from the past, present and future is, generally, a very constructive idea, which has been followed by many scholars. Without invoking physical laws, however, the philosophical program outlined by Reichenbach encounters mounting difficulties (briefly discussed below) and was not able to produce a transparent and concise representation of our temporal intuition in terms of objective casual connections.

\section{Universal causality}

Existence of causality in its broadest sense is often seen as being a most fundamental property of the universe, a priori postulate that does not need any further justification of explanation. As Bertrand Russell wrote in his book "Our knowledge of the external world" [2] 
The law of causation, according to which later events can theoretically be predicted by means of earlier events, has often been held to be a priori, a necessity of thought, a category without which science would not be possible.

We stress that this understanding of temporal causation, which we can call "universal causality", is a philosophical (rather than scientific) concept. We are interested only in causality associated with the direction of time (e.g. the past events causing the future events) and avoid any other interpretations of causality, which might also be important in philosophy. The domains of philosophy and science may overlap but, generally, do not coincide. The former reflects our most fundamental perceptions of reality, while the latter involves hypotheses and theories that can be tested experimentally and confirmed or invalidated. The principle of universal causality is beyond testable physical laws as these laws are deemed to be based on this principle. This, however, does not imply that universal causality is unconditionally supported in philosophy and objected in physics (in fact the opposite might be more correct: many philosophers refer to physical laws to clarify the concept of causality, while physicists tend to use causality as intuitive a priori to explain physical laws). The intuition based on causality is deeply embedded into human consciousness and most scientists tend to follow this intuition. Philosopher Huw Price [4] writes:

I have been trying to correct a variety of common mistakes and misconceptions about time in contemporary physics - mistakes and misconceptions whose origins lie in the distorting influence of our own ordinary temporal perspective, and especially of the time asymmetry of that perspective.

As noted above, experimental validation of universal causality is conceptually problematic. Even defining the meaning of "something causing something else" without invoking various physical laws is not a simple matter. For example, consider two events shown in figure 1
A. the vase falls
B. the vase breaks into pieces

Assume that the floor is hard (so that A inevitably causes B) and there is no malicious hooligan who can break the vase by some other means (so that B is impossible without A). While our intuition unmistakably identifies $\mathrm{A}$ as the cause and $\mathrm{B}$ as an effect, this intuition is not easy to define at a general level without appealing to physical laws that, in fact, may be implicitly based on causality. Obviously A and B are linked to each other. There is, however, no logical flaw in identifying B as the cause and A as the effect despite the fact that this contradicts our intuition: given that the vase is shattered into pieces, it must have fallen from the table. This basic example illustrates difficulties in formulating a broad definition of causality that involves reversible, irreversible and stochastic processes of microscopic and macroscopic scales and agrees with our intuitive perception of time. Russell [2] noted that

The view that the law of causality itself is a priori cannot, I think, be maintained by anyone who realises what a complicated principle it is.

Even more recent publications [12,5] have not brought a complete resolution to this problem. One can, of course, invoke the second law of thermodynamics, which, for the case illustrated in figure 1, determines that state $\mathrm{A}$ of the vase must precede state $\mathrm{B}$. The cause and the effect can then be distinguished among casually connected events by their temporal precedence, defining the earlier event as the cause and the later event as the effect [12. Generally, this definition is reasonable but, in the present context, this leads us to a circular argument - the direction of time is determined by causality, while causality is defined by temporal precedence. Defining causality in terms of precedence is perfectly acceptable from the philosophical perspective, but this definition downgrades universal causality to being a consequence of temporal precedence, which, in its turn is constrained by the laws of physics (i.e. by the second law of thermodynamics). In this context, we can simply overlook temporal precedence and define, for related events A and B, a lower entropy state (state A) as the cause and a higher entropy state (state B) as the effect. It seems that linking causality to irreversibility has become commonly accepted in modern philosophy and is sometimes referred to as the "standard interpretation" [5. This leads us to a less universal interpretation of causality that is connected to the physical laws, instead of being an unquestionable foundation of these laws. 
Some recent experiments [13, which are based on Wheeler's idea of delayed choice in quantum measurements, are sometimes seen as action of retro-causality in the quantum world. Considering the difficulty of finding a rigorous and comprehensive definition of causality, these experiments can only indicate that the concept of causality is less useful in interpretation of the microworld than in interpretation of the macroworld and, perhaps, less universal than it was previously thought. While results of these experiments may be alarming for proponents of universal causality, the usefulness of a more practical understanding of causality is not likely to be affected.

\section{4 "Practical" causality}

Consider an engineer who needs to simulate an industrial process over a given time interval. The engineer is hesitant whether he/she should set initial or final conditions for the process. Based on his/her intuition or explicitly using the causality principle (the future is determined by the past) the engineer decides to set the initial conditions and is, generally, absolutely right to do this. The engineer's concern is to obtain the most accurate simulations with minimal effort while bypassing complicated problems related to the nature of time. Invoking temporal causality and setting the initial conditions is, typically, the shortest path to practical success. The causality principle works quite well in most practical cases, replacing complex analysis of the properties of time by a simple rule of thumb: giving preference to the initial conditions over the final conditions.

There might be some cases when any type of conditions (initial or final) would allow us to perform the simulations equally well. The problem of elastic collisions of two particles may serve as an example. There are also cases when neither condition can guarantee a success. For example, evolution of a complex stochastic system is not well-defined by either initial or final conditions. However, the engineer's decision is absolutely reasonable, as practical cases in which setting the final conditions is better than setting the initial conditions are hard to find. Let us consider few examples of such rare cases. 1) It is easier to use the final conditions than initial conditions in the process of deciphering a message, 2) determining the path of a tornado might be easier using final conditions when the swath of plants and constructions destroyed by the tornado is known and 3) evaluating an experiment is easier after its completion, when the experimental records are available. We note that in all of these cases the apparent entropy associated with the information of interest is reduced forward in time (which is, of course, compensated by a large increase of entropy somewhere else). The last example involves continuous human interference recording most valuable experimental information; its complete simulation may need a combination of the initial conditions (i.e. the experimental set up) and final conditions (i.e. the recorded results).

Practical causality has a broad range of applications. Engineering is mentioned to emphasise the applied character of the interpretation of causality considered in this section. Besides engineering, this interpretation is also used commonly and effectively in various fields of science. The intuitive use of causality is justified as long as we do not need to dwell on investigating the fundamental properties of time and explain causality instead of presuming it - practical causality is a very useful concept and, generally, it is consistent with our experiences.

\section{Boltzmann's time hypothesis}

The arguments of the previous sections, outlining the utility of practical causality and difficulties of interpreting causality as a priori standing above and beyond physical laws, lead us to declare an epistemological connection between causality and the laws of physics. This section makes the next logical step, adding a direct physical relation to the previously declared connection: causality is to be understood as a consequence the physical laws and, hopefully, can be tested in conjunction with these laws.

While the directional nature of time needs to be related to measurable physical quantities, establishing this relation seems problematic - all major physical theories, including classical and quantum mechanics, special and general relativity are time-symmetric. There is an important exception represented by thermodynamics: entropy tends to increase forward in time according to the second law of thermodynamics. As previously noted, the directional properties of the second law have been repeatedly used by philosophers to define the preferential direction of time 12. This treatment, however, does not necessarily represent a physical hypothesis, which requires not only correspondence but also physical equivalence of the perceived flow of time and the direction of entropy increase. 
The physical time hypothesis was introduced by Boltzmann, who explains in his "Lectures on gas theory" [1]:

However, just as at a particular place on the earth's surface we call "down" the direction toward the center of the earth, so will a living being in a particular time interval of such a single world distinguish the direction of time toward the less probable [i.e. lower entropy $-\mathrm{AYK}$ ] state from the opposite direction (the former toward the past, the latter toward the future).

In his remarkable insight, Boltzmann sees the perceived direction of time as being physically determined by the temporal direction of the entropy increase: if entropy had to decrease in some segments of the universe, people living there would see our future as their past and our past as their future. Reichenbach [3] and Hawking [9] believe that the thermodynamic interpretation of the arrow of time explains existence of the psychological time arrow: we remember the past but do not remember the future. This point can be illustrated by the example "footsteps on a beach" shown in figure 2 - do these footsteps indicate that someone has walked on the beach in the past or that someone will walk on the beach in the future? While the answer is obvious to everyone, the explanation may appear a bit more subtle. The footsteps reflect the past due to action of the second law of thermodynamics: these footsteps can disappear without a cause due to increasing entropy erasing information (solid blue lines in figure 2), but they cannot appear without a cause (i.e. without someone walking on the beach), as this would contradict to the second law. Footsteps on a beach can be washed out but cannot be "washed in". The scenario outlined by the red dashed line in figure 2 contradicts to the second law and is impossible. Overall, the cause (a man walking) must precede the effect (footsteps on the sand) due to constraints of the second law.

A photograph or something that we simply remember are subject to the same constraints as the footsteps on a beach - they are governed by the second law and thus tell us about the past and not about the future. In the same way, the other observed arrows of time must reflect the thermodynamic arrow: surface waves cannot appear without being caused by a stone thrown into water and electromagnetic waves cannot appear without a prior electric impulse, but all these waves do not need any specific cause in the future and can gradually dissipate forward in time due to the action of the second law.

The example shown in figure 2 illustrates that the second law implies relative freedom in setting initial conditions by, say, walking on the beach (although this freedom is not absolute) as compared to much lesser freedom for achieving specific final conditions. The second law also provides a transparent explanation for the practical version of the causality principle. Consider two initial states A and A' of a thermodynamic system at $t=t_{1}$ that result in essentially the same equilibrium state $\mathrm{B}$ at $t=t_{2}$ (the phase volumes corresponding to these states are schematically shown in figure 3 ). The evolutions $\mathrm{A} \rightarrow \mathrm{B}$ and $\mathrm{A}^{\prime} \rightarrow \mathrm{B}$ can be simulated by setting the initial conditions at $t=t_{1}$ but not by setting the final conditions at $t=t_{2}$. Action of the second law of thermodynamics, which erases plenty of macroscopically significant information, commonly makes setting the final conditions ill-posed. Our preference for setting initial (and not final) conditions, which is innately interpreted as the past causing the future, is thus a natural outcome of the second law.

\section{Why does entropy increase?}

While the Boltzmann time hypothesis declares physical equivalence of the causal and thermodynamic arrows of time, the mechanism behind the action of the second law remains elusive. A number of factors (a few of these were originally considered by Boltzmann [14]) are commonly expected to be relevant to this mechanism. These factors are discussed in this section.

\subsection{Fixing the initial conditions}

The most common approach for demonstrating that entropy tends to increase in time is enforcing specific initial conditions and leaving the final conditions unconstrained. This may be perfectly satisfactory from the perspective of statistical physics, but does not achieve our goal since setting initial (as opposite to final) conditions presumes causality and implicitly discriminates the directions of time. One cannot explain causality in line with the Boltzmann time hypothesis by entropy increases and, at the same time, deduce the propensity of the entropy to increase in time from causality. The directions of time are often 
discriminated by very strong intuitive perception of the "time flow" based on causality, which is common among all people including scientists.

\subsection{The beginning and the end of the Universe}

The second law of the thermodynamics may be related to the expected temporal boundary conditions imposed on the Universe: low entropy at the beginning (i.e. uniform Big Bang) and, presumably, high entropy at the end [7. While such boundary conditions should undoubtedly affect global properties of entropy, the ubiquitous action of the second law raises the question about the mechanism that brings the global boundary conditions into every macroscopic process. Indeed, low initial and large final values of entropy in the universe explain the overall entropy-increasing trend but, on its own, cannot explain necessity of this increase in every experiment where measures are taken to screen the experiment from the initial and final conditions imposed on the universe. This, of course, does not preclude these initial and final conditions to act through some mechanisms that are not precisely known at present. Note that, in the present consideration, we must avoid the trap of a priori discrimination of the directions of time by presuming causality and setting only the low-entropy initial conditions.

Consider the example shown in figure 4; a system, which includes a Carnot engine with two heat reservoirs - the heater and the cooler - and a work reservoir [15], is held in a remote location of the universe. The engine preforms only two cycles (first, a cooling cycle and then the corresponding work-producing cycle) and is in a dormant state for thousand years before and after the cycles. In the dormant state, the reservoirs and the engine working gas are fully isolated from each other and are at their internal equilibria (or the there is a full equilibrium and temperature difference between the hot and cold reservoirs is created by the cooling cycle). Working of ideal Carnot cycles is reversible. This means that if the engine performs a cooling cycle forward in time, it is seen exactly as a work-producing cycle backward in time and vice versa. Observing these ideal cycles does not reveal the direction of time - even with the most detailed specification of the experimental parameters, we cannot tell if we are observing the cycles forward in time or backward in time.

The same experiment, however, would easily detect the action of the second law if conducted with more realistic cycles: both the cooling cycle and the work-producing cycle always underperform forward in time and overperform backward in time. If the laws of physics are completely time-symmetric, it must be the initial and final conditions imposed on the universe that interfere with the operation of our Carnot engine. Prior to and after the sequence of two cycles, the system remains statistically steady for thousand years and, during these periods, even the full record of all molecular motions remains timesymmetric. How can the system still "remember" the initial state imposed on the universe? There is no obvious answer. There might be a special field that is omnipresent everywhere and interferes with the system, connecting it to numerous ongoing time-directional processes in the rest of the universe. Another possibility is that the matter, which was created in the early universe and presently forms the parts of the engine, still "remembers" the directions of time (that is, the properties of matter are not fully time-symmetric due to special conditions in the early universe). In any case, there must be a specific mechanism affecting performance of the system in a time-directional manner and it should be possible, at least in principle, to detect this mechanism in experiments.

\subsection{Stosszahlansatz}

In his famous H-theorem, Boltzmann [1] demonstrated that the hypothesis of molecular chaos, called the Stosszahlansatz, is sufficient to ensure entropy increase in gases. This hypothesis discriminates the directions of time assuming that the properties of colliding particles (i.e. molecules) are statistically independent before (but not after) the collision. Abe [16] introduced a more general version of the Stosszahlansatz applicable to non-extensive thermodynamic systems. The Stosszahlansatz may have wider implications of a more generic statement: outputs following an event tend to correlate more than the inputs preceding this event. For example, scratches and dents on two cars have common patterns only after their collision and not before. Price [4] often uses term "innocence" to characterise independence of incoming inputs. Despite its importance and plausibility, the Stosszahlansatz (both in its original and more general forms) largely remains a hypothesis rather than a proven statement. Physically, the Stosszahlansatz, which is strongly time-directional, is likely to be related to time-directional properties of mixing, which are discussed below. 


\subsection{Ergodic mixing in classical systems}

From the classical perspective, thermodynamic systems can be seen as dynamic Hamiltonian systems of a very large dimension. As shown in figure $5 \mathrm{a}$, evolution of these systems is time-reversible, preserves volumes in the phase space (and, consequently, entropy, which is a logarithm of the phase volume), and is expected to uphold a special mixing property, which is often called ergodic mixing [17. This property requires that overwhelming majority of dynamic trajectories occupy the phase space with uniform average density irrespective of the initial (or final) conditions imposed on the trajectories, provided these conditions are separated from the current moment by a sufficiently long time interval. Under these conditions any tiny coarsening of the trajectories results in the initial phase volume expanding to the whole phase volume available to the system (figure 5 ). This coarsening occurs forward in time resulting in entropy increase in this direction. Backward in time, the same process represents "thinning" of the trajectories, reducing the occupied phase volume and entropy. The rate of the phase volume increase is specified by the so called Kolmogorov-Sinai dynamic entropy, which is determined by dynamic properties of the system and by the presence (but generally not the magnitude and characteristic time) of the coarsening. While it is possible to explain average entropy increase in this model without coarsening by setting initial (and not final) low-entropy conditions, this explanation implicitly invokes causality - this, as previously noted, would result in circular logic in the present context.

\subsection{Quantum mixing: decoherence and collapses}

While the major equations of quantum mechanics are time-reversible and entropy-preserving, irreversible quantum effects are associated with quantum decoherence and collapses (see illustration in figure 6 and the Appendix). Since understanding of decoherence varies between publications, this term is understood here as conversion of pure quantum states into mixed quantum states irrespective of the underlying physical mechanisms. The details are discussed in the Appendix. Introduction of decoherence replaces reversible unitary evolutions of the Schrödinger equation by the irreversible evolutions of the Pauli master equation [18, 8, 19. While conventional unitary evolutions of quantum systems preserve entropy, decoherence increases entropy and the maximal possible entropy is achieved in the maximally mixed state. Backward in time, decoherence is interpreted as recoherence, which removes uncertainty from the system and reduces entropy. As in the classical case, the characteristic time of decoherence $\tau_{d}$ does not affect the rate of entropy change (assuming that $\tau_{d}$ stays within its prescribed physical range). This rate is determined by the Hamiltonian specifying unitary interactions between major energy eigenstates. Nevertheless, seemingly minor alteration of the time symmetry by decoherence has a dramatic effect on the universe, introducing the arrow of time in kinetic equations [19, 20].

Decoherence may involve both spontaneous (intrinsic) and environmental mechanisms. The recent theories of decoherence tend to focus on decoherence induced by unitary interactions with the environment 21, 22, 23]. While environmental mechanisms can be expected to play a significant role in decoherence, the conceptual treatment of these interactions is based on unitary models, which cannot serve as explanations for the time flow. Indeed, any theories that are based on time-symmetric quantum mechanics rely on setting special initial conditions for introducing temporal directionality, and this implicitly invokes causality. These theories of environmental decoherence are useful in many respects but cannot explain the arrow of time. At the same time, many theories of spontaneous decoherence 24, 25, 26, which introduce time-directional modifications of the unitary quantum mechanics and, in principle, may explain the arrow of time, often lack physical interpretation and experimental validation.

In terms of the governing equations, decoherence and collapse replace the von Neumann equation for density matrices by the Lindblad equation (see the Appendix). The former preserves the entropy, while the latter changes entropy but preserves the trace and positiveness of the density matrix [8]. The Lindblad equation is more a framework for analysis rather than a specific model. However, many specific models of decoherence and collapse have been built around the Lindblad equation and a few of these models introduce stochastic interference with quantum systems (e.g. Refs. 27, 8, 25, 26, 28]). Most of these models are phenomenological: i.e. they may predict how decoherence acts but cannot explain why. 


\section{The time primer}

The Boltzmann time hypothesis establishes a fundamental link between the time arrow and entropy increase but, in its original form, does not represent an experimentally testable theory, since the second law of thermodynamics remains largely empirical and derived from engineering practice and understanding. The missing physical explanation for the directionality of the second law can be sought in statistical physics but, as discussed in the previous section, this approach has to face significant obstacles. Statistical physics incorporates classical and quantum mechanics but, ultimately, has to deal with the original problem - describing the time-asymetric real world while using time-symmetric mechanical laws. The existing approaches to justifying the second law of thermodynamics by presuming causality can only lead us to circular arguments.

At this point we assume existence of a "time primer" — a physical mechanism (or mechanisms) that enacts irreversible processes associated with the arrow of time (such as entropy increase, practical causality, decoherence, collapse, etc. ). In simple terms we do not state that the observed "time flow" is induced by the second law but rather that both the "time flow" and the second law have a common physical cause, which is not exactly known to modern science. The time primer may involve environmental and spontaneous (intrinsic) mechanisms, interactions, collapses and influences of the global boundary conditions - we do not restrict its mechanisms but expect that the physical action of the time primer can be tested experimentally, at least in principle. It seems that the most immediate measurable effect of the time primer should be quantum decoherence (i.e. the time primer must at least provide a reasonable physical explanation for quantum decoherence without presuming causality). In context of environmental mechanisms, only those environmental interactions that must be present intrinsically for enacting the direction of time are related to the time primer. As the direction of time seems to be universal in the world we know, the time primer is expected to be intrinsic or, at least, intrinsically present.

The existence of the time primer is a logical extension of the Boltzmann time hypothesis allowing us to avoid circular arguments, which are implicitly based on causality and were occasionally used by Boltzmann to explain the action of the second law. These circular arguments have only become more common in recent times [4. The time primer presumes the primacy of testable physical laws over causality, which is seen more as a practical consequence of these laws than a priori universal principle. Without specification of a particular mechanism, the time primer is nothing more than a place holder for a physical theory of thermodynamic time. Its role is in breaking circular arguments and becoming a "known unknown" rather than an "unknown unknown". The time primer allows us to discuss physical mechanisms enacting thermodynamic time without subscribing for a particular model of decoherence or any other related physical process. The possible mechanisms enacting the time primer may range from temporal asymmetries hidden inside baryons to the action of gravity.

\subsection{Properties of the time primer}

While the time primer is an assumption that the mechanism enacting the second law of thermodynamics and the perceived flow of time can be explained by a physical, experimentally testable theory (which remains unknown at present), we still can restrict properties of the time primer by applying constraints based on common knowledge. The time primer must be time-directional but should have a microscopic process of a very small magnitude, which is difficult (but ultimately possible) to detect, since the key time-symmetric theories of physics (e.g. classical and quantum mechanics, special and general relativity) produce reasonably accurate descriptions of reality. The time primer may reflect inherent properties of matter or be a result of spontaneous breaking of time symmetry. A theory explaining the time primer should not use causality as one of its postulates and discriminate the directions of time by merely setting the initial (and not final) conditions since, as previously noted, this would lead us to circular reasoning.

Generally, we should expect time priming to involve both environmental and spontaneous mechanisms, at least because distinguishing these mechanisms may not always be possible. Consider a thermodynamic system, which is located in a remote part of the universe in a way that prevents common thermodynamic interactions with the universe. Should thermodynamic time stop for this system? There is no evidence that this would be the case - the pace of irreversibly running time does not seem to depend on surroundings (for example, the rate of nuclear decays does not seem to slow down when environmental interference is reduced). Hence, if the universe still influences the system, enforcing running thermodynamic time, this should be some unavoidable interference, not constrained by locality and known interaction mechanisms. 
Philosophically, this type of unavoidable interference, which involves properties of the universe as the whole, is not much different from intrinsic behaviour associated with the system. We call this interference intrinsically present. After all, extending the concept of environmental interference from specific measurable interactions with devices and immediate environment to abstract remote interferences with the whole of the universe removes the line of separation between environmental and spontaneous mechanisms.

\subsection{Invariance of the time primer}

Since the time primer is expected to be a microscopic process violating time symmetry, the invariant properties of this process represent a key question. Presuming that matter and antimatter are fundamentally similar leads to two possibilities:

1. the time primer is $\mathrm{CP}$-preserving and CPT-violating or

2. the time primer is CPT-preserving and CP-violating.

The first possibility corresponds to the symmetric (CP-invariant) extension of thermodynamics from matter to antimatter, while the second possibility corresponds to the alternative antisymmetric (CPTinvariant) extension [29, 19]. As discussed in the rest of this work, these alternatives do have implications for our understanding of the properties of the universe. Note that the invariant properties of the time primer (i.e. $\mathrm{CP}$ or $\mathrm{CPT}$ ) are not necessarily determined by the invariant (CP or $\mathrm{CPT}$ ) properties of the unitary Hamiltonians [19.

\subsection{Diosi-Penrose theory}

In this subsection we consider a possible mechanism for time priming by gravitational collapse of quantum superposition states suggested by Diosi and Penrose [30, 31, 25, 32]. The physical substance of the theory, which pertains to interactions of gravity and quantum mechanics and distinguishes the DiosiPenrose model from other, more phenomenological approaches, is best explained by Penrose [31, 32, who discusses the fundamental difficulties of unifying quantum mechanics with general relativity. Quantum superpositions of masses should lead to superpositions of space-time geometries, which should redefine understanding of space and time accepted in general relativity and, according to Penrose [31, 32, lead to non-linear interactions of superimposed states making these states unstable. If $\Delta E$ is the self-gravitational energy associated with the superposition states, than $\tau \sim \hbar / \Delta E$ would be characteristic life time of these states, which becomes very small for massive objects. Hence, quantum superposition states are not observed for macroscopic objects - Schrödinger's cat must be either dead or alive. Despite the extremely small amplitude of self-gravity, there can be fine physical experiments that can detect existence of this mechanism, at least in principle [32].

While the Diosi-Penrose theory seems to be closer than any other model to discovering the physical mechanism behind the directionality of time, a number of questions remain unanswered. First, both general relativity and quantum mechanics are time-symmetric theories and it is not clear which effect can discriminate the directions of time. Would this be a spontaneous breaking of the time symmetry? If this is the case, why does time run in the same direction everywhere? Penrose's analysis [31, 32 implicitly invokes causality and does not answer these questions. Second, is the envisaged unified theory of quantum gravity expected to preserve the CPT invariance of relativistic quantum mechanics? A negative answer would deliver a devastating blow to our current understanding of the quantum world, which is inherently linked to Feynman's interpretation of antiparticles as particle equivalents moving backward in time.

\section{Detecting time priming in experiments}

\subsection{Decoherence experiments}

Intensive experimental investigation of decoherence started only recently and has been reviewed by Schlosshauer [33. The successful experiments can be divided into three groups: decoherence of qubit superposition states in superconductors [34, multi-slit wave interference for macro-molecules (with masses on the order of a thousand of atomic mass units) 35. and decoherence of photonic coherent states in a 
cavity [36]. It seems that all these experiments are dominated by environmental decoherence. The macromolecule interference picture improves with decreased gas pressures [37, clearly indicating interactions of the macro-molecules and the gas. Decoherence times of $20 \mathrm{~ns}$ (later of several $\mu \mathrm{s}$ [33], while qubits can be stored in semiconductors for more than 30mins [38) have been achieved in superconductors [34. The characteristic times of decoherence for photons in a cavity reach tens of ms and seem to be inversely proportional to the number of photons [36]. This, however, is likely to reflect bosonic interaction terms rather than any spontaneous behaviour - the number of photons in the experiment (tens) is far too small while spontaneous decoherence can be expected to become detectable only in larger systems.

While known experiments are likely to be dominated by environmental decoherence [33, this does not mean that spontaneous decoherence is not important, since the time primer, which determines the direction of time, may have a very small magnitude and produce only a small contribution in the experiments. To detect spontaneous mechanisms of decoherence, we need to reduce the influence of the environment to a minimum and increase the size of the system. We also note that some environmental interferences may be intrinsically present and thus practically indistinguishable from spontaneous mechanisms. The conventional wisdom of achieving sufficient system sizes for detecting spontaneous decoherence has nevertheless been questioned in some publications [39] under assumptions of decoherence driven by a stochastic interference. Penrose [32] describes experimental setup developed by Dirk Bouwmeeste that may be able to detect spontaneous decoherence predicted by Diosi-Penrose theory with the use of a photon reflection from a tiny mirror. While experimental validation of a physical theory, such as the one suggested by Diosi and Penrose, may be difficult but ultimately possible, a more generic examination physical nature of intrinsic decoherence is more problematic due to absence of any prior information about the possible magnitude of the process. Generally, it is impossible to search for something that has an unknown magnitude and mechanism, but there might be a possibility of detecting an unknown time primer due to its expected invariant properties.

\subsection{Detection of the environmental time priming in CP-violating processes}

Consider a quantum system in an environment with the overall Hamiltonian $\mathbf{H}_{U}$ represented in the Hilbert space $\mathcal{H}_{U}=\mathcal{H} \otimes \mathcal{H}_{E}$ by

$$
\mathbf{H}_{U}=\underbrace{\mathbf{H}_{s} \otimes \mathbf{I}_{E}}_{\sim 1}+\underbrace{\mathbf{H}_{w} \otimes \mathbf{I}_{E}}_{\sim \varepsilon}+\underbrace{\mathbf{I} \otimes \mathbf{H}_{E}}_{\sim 1}+\underbrace{\mathbf{H}_{\mathrm{int}}}_{\sim \varepsilon}
$$

where I denote the corresponding unit operators, the subscript " $E$ " refers to the environment, the term $\mathbf{H}_{\text {int }}$ reflects interaction of the system and environment. The state of the system and the environment in space $\mathcal{H}_{U}$ is specified by the orthogonal basis $|s\rangle \otimes|\beta\rangle$ denoted simply by $|s\rangle|\beta\rangle$. Here, $s=1, \ldots, n$ enumerates the states of the system and $\beta=1, \ldots, n_{E}$ represents a very large number of states in the environment. In many decaying systems (such as mesons), the system Hamiltonian has two components the larger $\mathbf{H}_{s}$, which is associated with the strong force and dominates the Hamiltonian, and $\mathbf{H}_{w}$, which is associated with the weak force and is responsible for deviations from strong eigenstates causing decays. The states of the system $s=\{k, f\}$ are divided into initial states $k$ and the final (or intermediate) states $f$. Note that $\left\langle k\left|\mathbf{H}_{s}\right| f\right\rangle=0=\left\langle f\left|\mathbf{H}_{s}\right| k\right\rangle$ but $\left\langle k\left|\mathbf{H}_{w}\right| f\right\rangle \neq 0 \neq\left\langle f\left|\mathbf{H}_{w}\right| k\right\rangle$. The small parameter $\varepsilon \ll 1$ indicates that the weak and environmental interaction terms are both small compared the strong force. While analyses neglecting environmental interactions in (1) are common [10, we can argue that unavoidable environmental influence must be present in these experiments. Indeed, particle decays are fundamentally irreversible, while unitary evolutions of the isolated quantum system are cyclic and eventually must return to original states. Hence time priming must be intrinsically present in decays. As discussed in the Appendix, irreversibility can be introduced through spontaneous or environmental decoherence. The former involves violations of quantum mechanics, while the latter requires consideration of interactions with the environment.

The suggested method of detecting unavoidable environmental interactions is based on the following key assumptions: the system under consideration is CPT-preserving (and may or may not be CPviolating), while interactions with the environment are CP-preserving. Specifically, $\mathbf{H}_{s}$ is both CPTand CP-preserving and $\mathbf{H}_{w}$ is CPT-preserving and can be CP-violating. The interactions with environment are assumed to be CP-preserving and time-assymetric. The assumption of CP-invariance of interactions with the environment is natural since overwhelming majority of the physical processes in the universe are CP-invariant. In combination with the temporal asymmetry of interactions with the 
environment, this may produce an impression of a CPT-violation, although this is not the case. Indeed, our treatment does not replace matter by antimatter in the environment (which is obviously impossible) and this makes charge conjugation $\mathrm{C}$ incomplete. Assuming that the composition of decaying particles is matter/antimatter symmetric, such as in $K$-mesons whose most stable configurations are approximately given by

$$
\left|K_{S}\right\rangle \approx \frac{|K\rangle+|\bar{K}\rangle}{\sqrt{2}}, \quad\left|K_{L}\right\rangle \approx \frac{|K\rangle-|\bar{K}\rangle}{\sqrt{2}}
$$

introducing time priming by spontaneous decoherence would violate the presumed CPT-invariance of the system. Hence, only environmental mechanism of decoherence can be consistent with the key assumptions listed above. Here, the initial states $k$ take two values: the kaon $|K\rangle$ and the antikaon $|\bar{K}\rangle$.

The problem can be solved by seeking an asymptotic solution for effective reduced Hamiltonian $\mathbf{H}_{\text {eff }}$ that acts in the space $|k\rangle|\beta\rangle$ in form of the series $\mathbf{H}_{\text {eff }}=\mathbf{H}_{0}+\varepsilon \mathbf{H}_{1}+\varepsilon^{2} \mathbf{H}_{2}+\ldots$, where $\left\langle k^{\prime}\left|\mathbf{H}_{0}\right| k^{\prime \prime}\right\rangle=$ $\left\langle k^{\prime}\left|\mathbf{H}_{s} \otimes \mathbf{I}_{E}\right| k^{\prime \prime}\right\rangle$ for $k^{\prime}, k^{\prime \prime}=K, \bar{K}$ while the other terms are evaluated with the Weisskopf-Wigner approximation [40. The possible CPT violation, which is indicated by the parameter

$$
\Lambda(\beta)=\left\langle K\left|\left\langle\beta\left|\mathbf{H}_{\text {eff }}\right| K\right\rangle\right| \beta\right\rangle-\left\langle\bar{K}\left|\left\langle\beta\left|\mathbf{H}_{\text {eff }}\right| \bar{K}\right\rangle\right| \beta\right\rangle
$$

appears only at the second order $\sim \varepsilon^{2}$ of the expansion:

$$
\Lambda(\beta)=-\sum_{f}\left(\left(\left\langle K\left|\left\langle\beta\left|\mathbf{H}_{\mathrm{int}}\right| f\right\rangle\right| \beta\right\rangle-\left\langle f\left|\left\langle\beta\left|\mathbf{H}_{\mathrm{int}}\right| K\right\rangle\right| \beta\right\rangle\right) \frac{\left\langle K\left|\mathbf{H}_{w}\right| f\right\rangle-\left\langle f\left|\mathbf{H}_{w}\right| K\right\rangle}{E_{0}-E_{f}+i \delta}\right)
$$

The sign of $\delta \rightarrow 0$ is selected to obtain only decaying exponents. Equation (4) requires that $\mathbf{H}_{w}$ is CPT-preserving and $\mathbf{H}_{\mathrm{int}}$ is CP-preserving. If both $\mathbf{H}_{w}$ and $\mathbf{H}_{\mathrm{int}}$ are CP-preserving, then $\Lambda(\beta)=0$ and there is no apparent CPT violation. Most quantum systems are CP-preserving, and for these systems, the invariant properties of weak and environmental interactions match each other so that the time priming by environmental interactions remains invisible. However, in the special cases of CP-violating systems, the environmental influence becomes visible as an apparent CPT violation $\Lambda(\beta) \neq 0[40$. This violation is only apparent and not real since the system is, intrinsically, CPT-preserving. Hence, it should be possible (at least in principle) to detect the unavoidable environmental mechanisms of time priming in CP-violating decays. Recent experiments seem to indicate that larger than expected CPT discrepancies are indeed present in CP-violating meson decays [10, 40, 41.

\subsection{Testing invariant properties of the spontaneous time primer}

At this point we return to consideration of spontaneous time priming. Detecting spontaneous time priming is always difficult. While this should be possible on the basis of a testing a specific theory or a mechanism (such as the theory by Diosi and Penrose), here we avoid specific assumptions about the mechanism of the time primer and ask only one question: is spontaneous time priming CP- or CPT-invariant? Since radiation must be decoherence neutral [20, spontaneous time priming needs to be associated with the most fundamental properties of matter and antimatter leaving us two possibilities: the directions of time priming for matter and antimatter are either the same or opposite. This gives rise to two alternative possible thermodynamics: symmetric and antisymmetric [29, 19, 20. This alternative can be tested experimentally — we only need to create a thermodynamic antisystem (i.e. a thermodynamic system made of antimatter) and screen it from the overriding thermodynamic influence of the environment (see Refs. [29, 19, 20] for details). If the antisymmetric thermodynamics is proven to be real, this would indicate the spontaneous mechanisms of time priming.

\section{Conclusions}

Universal causality, which for some time was presumed to be a priori of rational thinking about the universe, leads to a spectrum of unresolvable problems and has gradually been replaced by more practical interpretations of causality that does not stay above the physical laws but is linked to these laws or derived from them. In this context, Boltzmann's time hypothesis identifying the observed direction of time with the second law of thermodynamics seems most promising. We also expect that the action of the second law and the arrow of time are initiated by physical process of a very small scale that, at least in principle, 
can be detected in the experiments. This physical process is termed here as the "time primer". While the action of the time primer is extremely difficult to distinguish from other interactions, the recent works on experimental investigation of decoherence gradually progress in this direction. Besides these experiments, it seems that the intrinsically present action of the time primer can be detected in CP-violating (and CPT-preserving) decays of elementary particles and should be seen as apparent CPT violations.

\section{References}

[1] L. Boltzmann. Lecures on gas thoery. English translation by S.G. Brush. University of California Press, Berkeley and L.A., 1964 (1895,1897).

[2] B. Russell. Our Knowledge of the External World. Taylor and Francis, Florence, 2009.

[3] H. Reichenbach. The direction of time. University of California Press, Berkeley, 1971.

[4] H. Price. Time's Arrow and Archimedes' Point: New Directions for the Physics of Time. Oxford Univ. Press, Oxford, UK, 1996.

[5] J. Faye, U. Scheffler, and M. Urchs. Perspectives on time. Boston studies in the philosophy of science ; v. 189. Kluwer Academic Publishers, Dordrecht ; Boston, 1997.

[6] I. Prigogine. From being to becoming : time and complexity in the physical sciences. W. H. Freeman, San Francisco, 1980.

[7] R. Penrose. Road to Reality: A Complete Guide to the Laws of the Universe. A. Knopf Inc., 2005.

[8] H. D. Zeh. The physical basis of the direction of time. Springer, New York;Berlin;, 5th edition, 2007.

[9] S. W. Hawking. A brief history of time : from the big bang to black holes. Bantam, London, 2011.

[10] Beringer J. et al. (Particle Data Group). The review of particle physics. Phys. Rev., D 86:010001, 2012.

[11] P. Zwart. The flow of time. Synthese, 24(1):133-158, 1972.

[12] P. Dowe. Process causality and asymmetry. Erkenntnis, 37(2):179-196, 1992.

[13] A. G. Manning, R. I. Khakimov, R. G. Dall, and A. G. Truscott. Wheeler's delayed-choice gedanken experiment with a single atom. Nature Physics, 11(7), 2015.

[14] A. Y. Klimenko. What is mixing and can it be complex? Physica Scripta, T155:014047, 2013.

[15] E. P. Gyftopoulos and G. P. Beretta. Thermodynamics. Foundations and Applications. Macmillan, N.Y., USA, 1991.

[16] S. Abe. Generalized molecular chaos hypothesis and the h theorem: Problem of constraints and amendment of nonextensive statistical mechanics. Physical Review E, 79(4), 2009.

[17] J. L. Lebowitz and O. Penrose. Modern ergodic theory. Physics today, (Feb.):23-28, 1973.

[18] W. Pauli. Uber das h-theorem vom anwachsen der entropie vom standpunkt der neuen quantenmechanik. In Probleme der Modernen Physik. Arnold Sommerfeld zum 60 Geburtstage, pages 30-45. Hirzel, Leipzig, 1928.

[19] A. Y. Klimenko. Symmetric and antisymmetric forms of the pauli master equation. Scientific Reports (nature.com), 6:29942, 2016.

[20] A. Y. Klimenko. Kinetics of interactions of matter, antimatter and radiation consistent with antisymmetric (CPT-invariant) thermodynamics. 2017.

[21] W. H. Zurek. Decoherence, einselection, and the quantum origins of the classical. Reviews of Modern Physics, 75(3), 2003. 
[22] N. Linden, S. Popescu, A. J. Short, and A. Winter. Quantum mechanical evolution towards thermal equilibrium. Phys. Rev. E, 79:061103, 2009.

[23] V.I. Yukalov. Equilibration and thermalization in finite quantum systems. arXiv:1201.2781, 2012.

[24] A. Bassia and G. Ghirardi. Dynamical reduction models. Physics Reports., 379:257-426, 2003.

[25] L. Diosi. Intrinsic time-uncertainties and decoherence: comparison of 4 models. Brazilian Journal of Physics, 35(2a), 2005.

[26] S. Cano-Andrade, G. P. Beretta, and M. R. Von Spakovsky. Steepest-entropy-ascent quantum thermodynamic modeling of decoherence in two different microscopic composite systems. Physical Review A, 91(1), January 2015.

[27] L. Diosi. Models for universal reduction of macroscopic quantum fluctuations. Physical Review A, 40(3):1165-1174, 1989.

[28] C. Ou, R. V. Chamberlin, and S. Abe. Lindbladian operators, von neumann entropy and energy conservation in time-dependent quantum open systems. Physica A: Statistical Mechanics and its Applications, 466:450-454, 2017.

[29] A. Y. Klimenko and U. Maas. One antimatter- two possible thermodynamics. Entropy, 16(3):11911210, 2014.

[30] L. Diosi. A universal master equation for the gravitational violation of quantum mechanics. Physics Letters A, 120(8):377-381, 1987.

[31] R. Penrose. On gravity's role in quantum state reduction. General Relativity and Gravitation, 28(5):581-600, 1996.

[32] R. Penrose. On the gravitization of quantum mechanics 1: Quantum state reduction. Foundations of Physics, 44(5):557-575, 2014.

[33] M. A. Schlosshauer. Decoherence and the quantum-to-classical transition. The frontiers collection. Springer, Berlin ; London, 2007.

[34] I. Chiorescu, Y. Nakamura, C. Harmans, and J. Mooij. Coherent quantum dynamics of a superconducting flux qubit. Science, 299(5614):1869-1871, 2003.

[35] L. Hackermller, K. Hornberger, B. Brezger, A. Zeilinger, and M. Arndt. Decoherence of matter waves by thermal emission of radiation. Nature, 427(6976), 2004.

[36] S. Delglise, I. Dotsenko, C. Sayrin, J. Bernu, M. Brune, J.-M. Raimond, and S. Haroche. Reconstruction of non-classical cavity field states with snapshots of their decoherence. Nature, 455(7212), 2008.

[37] L. Hackermller, K. Hornberger, B. Brezger, A. Zeilinger, and M. Arndt. Decoherence in a talbotlau interferometer: the influence of molecular scattering. Applied Physics B, 77(8):781-787, 2003.

[38] K. Saeedi, S. Simmons, J. Z. Salvail, P. Dluhy, H. Riemann, N. V. Abrosimov, P. Becker, H.-J. Pohl, J. J. L. Morton, and M. L. W. Thewalt. Room-temperature quantum bit storage exceeding 39 minutes using ionized donors in silicon-28. Science, 342(6160):830-833, 2013.

[39] S. Nimmrichter, K. Hornberger, and K. Hammerer. Optomechanical sensing of spontaneous wavefunction collapse. Physical review letters, 113(2):020405, 2014.

[40] A.Y. Klimenko. Note on invariant properties of a quantum system placed into thermodynamic environment. Physica A: Statistical Mechanics and its Applications, 398:65 - 75, 2014.

[41] Lees, J. P. et. al. (BABAR Collaboration). Tests of $c p t$ symmetry in $B^{0}-\bar{b}^{0}$ mixing and in $B^{0} \rightarrow c \bar{c} K^{0}$ decays. Phys. Rev. D, 94:011101, 2016. 
[42] K. Camilleri. A history of entanglement: Decoherence and the interpretation problem. Studies In History and Philosophy of Modern Physics, 40:290-302, 122009.

[43] P. C. E. Stamp. Environmental decoherence versus intrinsic decoherence. Philosophical transactions. Series A, Mathematical, physical, and engineering sciences, 370(1975):4429, 2012.

[44] W. H. Zurek. Environment-induced superselection rules. Phys. Rev. Lett., 26(8):1862-1888, 1982.

[45] E. Joos, C. Kiefer, and H. D. Zeh. Decoherence and the Appearance of a Classical World in Quantum Theory. Springer Berlin Heidelberg, Berlin, Heidelberg, 2 edition, 2003.

[46] John Von Neumann. Mathematical foundations of quantum mechanics. Investigations in physics ; 2. Princeton University Press, Princeton N.J., 1955.

[47] L. D. Landau and E. M. Lifshits. Course of Theoretical Physics vol.5: Statistical physics. Butterworth-Heinemann, Oxford, 1980.

[48] H. Everett. "relative state" formulation of quantum mechanics. Reviews of Modern Physics, 29(3):454-462, 1957.

[49] B. S. Dewitt. Quantum mechanics and reality. Physics Today, 23(9):30-35, 1970.

\section{A Appendix: decoherence and collapse}

The arrow of time is believed to be closely connected to irreversible processes of quantum mechanics 7. 8. While interpretation of these processes is a difficult problem on its own, the reader can be easily confused by inconsistencies of definitions used in different publications. For example, decoherence is often defined only as an environment-induced process, while spontaneous behaviour is related exclusively to quantum collapses [42]. Other publications (e.g. 43]), however, refer to both environmental and intrinsic decoherences. This appendix clarifies the use of the terms "decoherence" and "collapse" in the present context of considering the Boltzmann time hypothesis.

Restricting term "decoherence" only to environmental processes would be rather inconvenient, since both types of decoherence, environmental and spontaneous, produce very similar effects. In addition, both types of decoherence are likely to act in combination with each other and, as discussed in Section 8, are very difficult to distinguish experimentally. Decoherence is understood here is a process of converting pure quantum states into mixed states or increasing the degree of mixing in already mixed states, irrespective of the physical mechanism(s) responsible for the process. Decoherence increases the entropy $S$ of the system. Collapse is reduction of the wave function to a particular state during measurements or interactions with macroscopic objects and, besides decoherence, may involve other effects. If spontaneous decoherence is to be exclusively associated with collapse, the latter term needs further qualification, as discussed below. While the author of the present work tends to use the terms "spontaneous decoherence" and "intrinsic decoherence" synonymously, there is a semantic difference between these terms that is worth noting. "Spontaneous" means "without an external cause" while "intrinsic" implies impossibility to avoid something by changing external conditions. Hence, interactions of a quantum system with an omnipresent time-priming field (assuming that such a field exists) are intrinsic but not spontaneous.

\section{A.1 Modelling decoherence}

A general framework for consideration of models deviating from unitary evolution is given by the Lindblad equation

$$
\underbrace{i \hbar \frac{\partial \boldsymbol{\rho}}{\partial t}=[\mathbf{H}, \boldsymbol{\rho}]}_{\text {von Neumann Eq. }}-\underbrace{\frac{i}{2} \sum_{j=1}^{n^{2}-1}\left(\mathbf{L}_{j}^{\dagger} \mathbf{L}_{j} \boldsymbol{\rho}+\boldsymbol{\rho} \mathbf{L}_{j}^{\dagger} \mathbf{L}_{j}-2 \mathbf{L}_{j} \boldsymbol{\rho} \mathbf{L}_{j}^{\dagger}\right)}_{\text {Lindblad operator }}
$$

for density matrices $\boldsymbol{\rho}$ that are expressed by the sum of the outer products of the wave functions $\psi_{k}$

$$
\boldsymbol{\rho}=\sum_{k=1}^{N} p_{k}\left|\psi_{k}\right\rangle\left\langle\psi_{k}\right|
$$


The state specified by this equation is mixed if $N>1$ and pure if $N=1$. The mixture is maximally ranked if $N$ reaches the dimension of the system $N=n$. The state is maximally mixed if $N=n$ and $p_{k}=1 / n$ for $k=1,2, \ldots, n$. The maximally mixed state corresponds to microcanonical thermodynamic equilibrium in macroscopic objects, attaining the maximal value of the entropy $S$, which is defined in quantum mechanics as

$$
S=-k_{B} \operatorname{Tr}(\rho \ln (\boldsymbol{\rho})),
$$

where $k_{B}$ is the Boltzmann constant. The von Neumann equation for $\boldsymbol{\rho}$ corresponds to the Schrödinger equation for the wave functions $\psi_{k}$, which represents reversible, unitary evolution and preserves the entropy: $d S / d t=0$. The Lindblad operator in equation (5) introduces changes of entropy $d S / d t \neq 0$ but preserves the trace $\operatorname{Tr}(\boldsymbol{\rho})=1$ and positiveness of the density matrix $\boldsymbol{\rho}$. While the general form of equation (5) does not guarantee monotonic increase of the entropy, there would be many specific forms of this equation that ensure that $d S / d t \geq 0$ (e.g. [26, 28]).

The Lindblad equation can specify various non-unitary behavious including decoherence and collapse. Decoherence corresponds to disappearance of non-diagonal elements in the density matrix $\rho$ :

$$
\left[\begin{array}{ccc}
\rho_{11} & \cdots & \rho_{n 1} \\
\vdots & \ddots & \vdots \\
\rho_{1 n} & \cdots & \rho_{n n}
\end{array}\right] \longrightarrow\left[\begin{array}{ccc}
\rho_{11} & & 0 \\
& \ddots & \\
0 & & \rho_{n n}
\end{array}\right]
$$

If a system interacts with environment (whose Hilbert space is denoted by $\mathcal{H}_{E}$ ) so that the "universe" is characterised by a Hilbert space $\mathcal{H}_{U}=\mathcal{H} \otimes \mathcal{H}_{E}$, then the density matrix of the system $\boldsymbol{\rho}$ can be obtained from the density matrix of the universe $\boldsymbol{\rho}_{U}$ by tracing out the degrees of freedom associated with the environment

$$
\boldsymbol{\rho}=\operatorname{Tr}_{E}\left(\boldsymbol{\rho}_{U}\right)
$$

As it has been shown by Zurek [44, very weak interactions of the system with environment are sufficient to cause prompt decoherence of the system even if the process is controlled by the conventional Schrödinger equation. There are no doubts that environmental interpretation of the decoherence is attractive the complicated problem of modifying unitary dynamics in equation (5) is apparently avoided. The environmental approach to decoherence has been advocated by many [45, 33, 22, 23. and quite rightfully so. It is most likely that environmental decoherence dominates in many practical situations (see section 8). We, however, cannot forget that discarding intrinsic decoherence completely would lead our analysis into unresolvable problems. Indeed, presuming absence of any directional time-priming mechanism must be compensated by the de-facto introduction of causality and, as previously noted, this leads us to circular arguments. Zurek's [44 decoherence theory is necessarily based on causality. Another problem is that the entropy increase - a fundamental process that, according to Boltzmann's time hypothesis, has a determining influence on temporal properties of the universe - does not occur in the universe according to this model (any entropy increase in the system is compensated by exactly the same entropy decrease in the environment). Arguments based on treating the environment as an infinite sequence of nested environments (akin Russian dolls) may hide the entropy problem for the system but does not form a realistic model reflecting the overall entropy increase in the universe.

\section{A.2 Possible stages of collapse}

Quantum measurements and, in fact, many other forms interactions of quantum systems with macroscopic objects (such as Schrödinger's cat) lead to collapse of the wave function [7. As discussed below, this collapse is related to but is not fully synonymous to decoherence. In our analysis, we intend to save the cat's life and treat quantum/classic interactions as a measurement problem. We broadly follow von Neumann's theory of quantum measurement [46], but consider variations around the Copenhagen interpretation associated with decoherence.

0. Initial state. Initially, the quantum system is in a superposition state and the measurement apparatus is in the "ready" state $\left|a_{0}\right\rangle$. The corresponding wave functions of the system $\psi_{s}=c_{\uparrow}|\uparrow\rangle+c_{\downarrow}|\downarrow\rangle$ and the apparatus $\psi_{a}=\left|a_{0}\right\rangle$ indicate that the initial combined system-apparatus state is given by

$$
\Psi_{0}=\psi_{s} \otimes \psi_{a}=c_{\uparrow}|\uparrow\rangle\left|a_{0}\right\rangle+c_{\downarrow}|\downarrow\rangle\left|a_{0}\right\rangle
$$


Here, we assume for simplicity that the quantum system has only two states $|\uparrow\rangle$ and $|\downarrow\rangle$, but avoid the trivial cases of $c_{\uparrow}=0$ and $c_{\downarrow}=0$. All states are presumed orthogonal. The corresponding density matrix $\boldsymbol{\rho}_{0}=\left|\Psi_{0}\right\rangle\left\langle\Psi_{0}\right|$ is given by

$$
\rho_{0}=c_{\uparrow}^{*} c_{\uparrow}|\uparrow\rangle\left|a_{0}\right\rangle\left\langle\uparrow\left|\left\langle a_{0}\left|+c_{\downarrow}^{*} c_{\downarrow}\right| \downarrow\right\rangle\right| a_{0}\right\rangle\left\langle\downarrow\left|\left\langle a_{0}\left|+c_{\downarrow}^{*} c_{\uparrow}\right| \uparrow\right\rangle\right| a_{0}\right\rangle\left\langle\downarrow\left|\left\langle a_{0}\left|+c_{\uparrow}^{*} c_{\downarrow}\right| \downarrow\right\rangle\right| a_{0}\right\rangle\langle\uparrow|\left\langle a_{0}\right|
$$

where and the asterisk "*" denotes the complex conjugates. The initial superposition state corresponds to zero entropy

$$
S_{0}=-k_{B} \operatorname{Tr}\left(\boldsymbol{\rho}_{0} \ln \left(\boldsymbol{\rho}_{0}\right)\right)=0
$$

1. Unitary co-evolution. Interactions between the system and the apparatus result in a transformation $\Psi_{0} \longrightarrow \Psi_{1}$

$$
\Psi_{1}=c_{\uparrow}|\uparrow\rangle\left|a_{\uparrow}\right\rangle+c_{\downarrow}|\downarrow\rangle\left|a_{\downarrow}\right\rangle
$$

implying that the corresponding density matrix $\boldsymbol{\rho}_{1}=\left|\Psi_{1}\right\rangle\left\langle\Psi_{1}\right|$ is

$$
\boldsymbol{\rho}_{1}=c_{\uparrow}^{*} c_{\uparrow}|\uparrow\rangle\left|a_{\uparrow}\right\rangle\left\langle\uparrow\left|\left\langle a_{\uparrow}\left|+c_{\downarrow}^{*} c_{\downarrow}\right| \downarrow\right\rangle\right| a_{\downarrow}\right\rangle\left\langle\downarrow\left|\left\langle a_{\downarrow}\left|+c_{\downarrow}^{*} c_{\uparrow}\right| \uparrow\right\rangle\right| a_{\uparrow}\right\rangle\left\langle\downarrow\left|\left\langle a_{\downarrow}\left|+c_{\uparrow}^{*} c_{\downarrow}\right| \downarrow\right\rangle\right| a_{\downarrow}\right\rangle\langle\uparrow|\left\langle a_{\uparrow}\right|
$$

where the sates of apparatus $\left|a_{\uparrow}\right\rangle$ and $\left|a_{\downarrow}\right\rangle$ are interpreted as measuring $|\uparrow\rangle$ and measuring $|\downarrow\rangle$. This transformation is conventionally assumed unitary (more precisely, it can be made unitary by proper selection of responses to other initial states, say $|\uparrow\rangle\left|a_{\uparrow}\right\rangle$ or $|\uparrow\rangle\left|a_{\downarrow}\right\rangle$ that are not of interest here). The unitary transformation can be more complicated than specified by (13), as long as $\left|a_{\uparrow}\right\rangle \neq\left|a_{\downarrow}\right\rangle$ and the initial states of the system can be distinguished. Unitary evolutions do not change the value of the entropy

$$
S_{1}=-k_{B} \operatorname{Tr}\left(\boldsymbol{\rho}_{1} \ln \left(\boldsymbol{\rho}_{1}\right)\right)=0
$$

2. Decoherence. Interaction with the apparatus, which is a macroscopic object, involves another process $\Psi_{1} \longrightarrow \Psi_{2}$, which is conventionally called decoherence. This process is non-unitary and/or can involve environmental interference. According to Zurek's theory of decoherence [44, 21] even the smallest interactions of quantum systems with macroscopic objects that do not transfer any substantial energy can result in a loss of coherence, that is

$$
\Psi_{2}=\Theta_{\uparrow} c_{\uparrow}|\uparrow\rangle\left|a_{\uparrow}\right\rangle+\Theta_{\downarrow} c_{\downarrow}|\downarrow\rangle\left|a_{\downarrow}\right\rangle
$$

where $\left|\Theta_{k}\right|=1, k=\{\uparrow, \downarrow\}$ and the phases $\theta_{k}=\ln \left(\Theta_{k}\right) / i$ become unpredictable or, practically, random due to decohering interference of the system with macroscopic objects. As the result of decoherence, the density matrix becomes diagonal $\boldsymbol{\rho}_{1} \longrightarrow \boldsymbol{\rho}_{2}$ where

$$
\rho_{2}=\overline{\left|\Psi_{2}\right\rangle\left\langle\Psi_{2}\right|}=c_{\uparrow}^{*} c_{\uparrow}|\uparrow\rangle\left|a_{\uparrow}\right\rangle\left\langle\uparrow\left|\left\langle a_{\uparrow}\left|+c_{\downarrow}^{*} c_{\downarrow}\right| \downarrow\right\rangle\right| a_{\downarrow}\right\rangle\langle\downarrow|\left\langle a_{\downarrow}\right|
$$

and the off-diagonal terms disappear (or, depending on the interpretation, effectively disappear or disappear on average) due to the absence of the phase correlations $\overline{\Theta_{\uparrow}^{*} \Theta_{\downarrow}}=0$, where the overbar denotes averaging over the random values. The corresponding entropy increases $S_{1} \longrightarrow S_{2}$, where

$$
S_{2}=-k_{B} \operatorname{Tr}\left(\boldsymbol{\rho}_{2} \ln \left(\boldsymbol{\rho}_{2}\right)\right)=-k_{B}\left(p_{\uparrow} \ln p_{\uparrow}+p_{\downarrow} \ln p_{\downarrow}\right)>0,
$$

and $p_{\uparrow}=c_{\uparrow}^{*} c_{\uparrow}, p_{\downarrow}=c_{\downarrow}^{*} c_{\downarrow}$ are non-negative real numbers, satisfying $p_{\uparrow}+p_{\downarrow}=1$ due to normalisation of the wave function.

Our consideration needs some clarifications at this stage. Realistically, the apparatus is a macroscopic object. That is, in addition to the measurement states $\left|a_{k}\right\rangle=\left\{\left|a_{0}\right\rangle,\left|a_{\uparrow}\right\rangle,\left|a_{\downarrow}\right\rangle, \ldots\right\}$, the apparatus is characterised by a very large number $n$ of microscopic states $\left|q_{j}\right\rangle, j=1, \ldots, n$ so that the apparatus has, at least $3 n$ quantum states $\left|a_{k}\right\rangle\left|q_{j}\right\rangle$. Since the apparatus is macroscopic, its state cannot significantly deviate from the maximally mixed state, which represents a thermodynamic equilibrium under microcanonical conditions (for example, as a macroscopic object, the apparatus cannot be in a superposition state, say, $\psi_{a}=\Sigma_{j} q_{j}\left|a_{0}\right\rangle\left|q_{j}\right\rangle$ due to almost instant action of the time primer). Even very weak interactions of the quantum system with a macroscopic object can lead to very fast decoherence, whose joint effect with unitary evolution can be characterised by the transition $\boldsymbol{\rho}_{0} \longrightarrow \boldsymbol{\rho}_{2}$. The decoherence of the quantum system states is not spontaneous - it is induced by the measuring apparatus. As a macroscopic object, the apparatus is influenced by the time primer to stay close to its maximally mixed state. The question of whether this time primer is intrinsic to the apparatus or induced by the environment surrounding the apparatus (or both) does not affect our consideration. 
3. Latent collapse. The next presumed stage in quantum measurement is collapse of the wave function $\Psi_{2} \longrightarrow \Psi_{3}$ with the probability $P$ specified by the Born rule

$$
\Psi_{3}= \begin{cases}|\uparrow\rangle\left|a_{\uparrow}\right\rangle, \quad P=c_{\uparrow}^{*} c_{\uparrow}=p_{\uparrow} \\ |\downarrow\rangle\left|a_{\downarrow}\right\rangle, \quad P=c_{\downarrow}^{*} c_{\downarrow}=p_{\downarrow}\end{cases}
$$

We must emphasise that, at this stage, we do not know (and, as discussed below, cannot possibly know) the outcome of the measurement (i.e. $|\uparrow\rangle\left|a_{\uparrow}\right\rangle$ or $|\downarrow\rangle\left|a_{\downarrow}\right\rangle$ ), although the wave function $\Psi$ has already collapsed into one of the states $|\uparrow\rangle\left|a_{\uparrow}\right\rangle$ or $|\downarrow\rangle\left|a_{\downarrow}\right\rangle$. The density matrix is then represented by the densities of two states $\boldsymbol{\rho}_{\uparrow}=|\uparrow\rangle\left|a_{\uparrow}\right\rangle\langle\uparrow|\left\langle a_{\uparrow}\right|$ and $\boldsymbol{\rho}_{\downarrow}=\left|a_{\downarrow}\right\rangle\langle\downarrow|\left\langle a_{\downarrow}\right|$ taken with the corresponding probabilities $p_{\uparrow}$ and $p_{\downarrow}$ :

$$
\boldsymbol{\rho}_{3}=p_{\uparrow} \boldsymbol{\rho}_{\uparrow}+p_{\downarrow} \boldsymbol{\rho}_{\downarrow}=p_{\uparrow}|\uparrow\rangle\left|a_{\uparrow}\right\rangle\left\langle\uparrow\left|\left\langle a_{\uparrow}\left|+p_{\downarrow}\right| \downarrow\right\rangle\right| a_{\downarrow}\right\rangle\langle\downarrow|\left\langle a_{\downarrow}\right|
$$

Note that $\rho_{3}=\rho_{2}$ and

$$
S_{3}=-k_{B} \operatorname{Tr}\left(\boldsymbol{\rho}_{3} \ln \left(\boldsymbol{\rho}_{3}\right)\right)=S_{2}>0
$$

The reader may notice that there is only a fine logical line separating the states 2 and 3 . Practically, the outcomes of decoherence and latent collapse are very similar but, still, there is a difference. Indeed, in the state 2 , the uncertainty between states $|\uparrow\rangle$ and $|\downarrow\rangle$ is deeply embedded into physics of the system, while in the state 3 , the system is already in one of the states $|\uparrow\rangle$ or $|\downarrow\rangle$, we simply do not know which one is that. We can call randomness and associated probability ontic or aleatory (i.e. related to objective physical uncertainty) in the first case, and epistemic (i.e. related to lack of knowledge) in the second case. While the existence of intuitive and philosophical differences between ontic and epistemic interpretations of probability needs to be acknowledged, quantum mechanics tends to treat states 2 and 3 as physically equivalent (or, at least, equivalent for all practical purposes), and has good reasons for this. Indeed, no measurement of any observable physical quantity $A$ can possibly distinguish these two states since $\boldsymbol{\rho}_{3}=\boldsymbol{\rho}_{2}$ and $A=\operatorname{Tr}\left(\boldsymbol{\rho}_{2} \mathbf{A}\right)=\operatorname{Tr}\left(\boldsymbol{\rho}_{3} \mathbf{A}\right)$. The equivalence of ontic and epistemic probabilities assigns objective interpretation to epistemic probability in quantum measurements - the observer cannot obtain knowledge without interfering with the system. This leads us to conclusion that epistemic uncertainty is objectively present in the surrounding world since, under conditions of non-interference, it cannot be distinguished from "genuinely objective" ontic uncertainty. This approach is reasonable in application to quantum measurements but may cause unease for many people if it is interpreted as a general principle applicable to macroscopic world. Einstein's famous "I like to think the moon is there even if I am not looking at it" insists that physical reality is one thing, while knowing or not about this reality is another.

4. Observable collapse. This is the final stage of the measurement process, when the result of the measurement becomes known (or can become known) due to producing a macroscopic effect. This stage can lead to one of the two possible outcomes:

$$
\left.4^{\prime}\right) \quad \Psi_{4^{\prime}}=|\uparrow\rangle\left|a_{\uparrow}\right\rangle, \quad \rho_{4^{\prime}}=|\uparrow\rangle\left|a_{\uparrow}\right\rangle\langle\uparrow|\left\langle a_{\uparrow}\right|
$$

or

$$
\left.4^{\prime \prime}\right) \quad \Psi_{4^{\prime \prime}}=|\downarrow\rangle\left|a_{\downarrow}\right\rangle, \quad \boldsymbol{\rho}_{4^{\prime \prime}}=|\downarrow\rangle\left|a_{\downarrow}\right\rangle\langle\downarrow|\left\langle a_{\downarrow}\right|
$$

with the corresponding frequencies proportional to $p_{\uparrow}$ and $p_{\downarrow}$ in repeated experiments. The outcomes of the observed collapse are very different from the outcomes of decoherence. In both cases $4^{\prime}$ and $4^{\prime \prime}$ the entropy $S$ is zero

$$
S_{4^{\prime}}=-k_{B} \operatorname{Tr}\left(\boldsymbol{\rho}_{4^{\prime}} \ln \left(\boldsymbol{\rho}_{4^{\prime}}\right)\right)=0=-k_{B} \operatorname{Tr}\left(\boldsymbol{\rho}_{4^{\prime \prime}} \ln \left(\boldsymbol{\rho}_{4^{\prime \prime}}\right)\right)=S_{4^{\prime \prime}}
$$

This apparent reduction of entropy indicates that other factors must be present in observing the results of measurement. We may consider interference of an observer, which has two possible outcomes of the measurement: $|\uparrow\rangle\left|a_{\uparrow}\right\rangle\left|o_{\uparrow}\right\rangle$ or $|\downarrow\rangle\left|a_{\downarrow}\right\rangle\left|o_{\downarrow}\right\rangle$, where $\left|o_{\uparrow}\right\rangle$ and $\left|o_{\downarrow}\right\rangle$ correspond to "observing $\uparrow "$ and "observing $\downarrow "$. We will try, however, to keep not only cats but also humans out of the experiments (as both are known to have "bad" behaviours that are rather difficult to predict) and focus on the measuring apparatus as a thermodynamic object. 
Since, according to the second law, entropy cannot have a consistent unmitigated decrease $0<$ $S_{3} \longrightarrow S_{4}=0$ even by a small amount (fluctuations are possible, of course, but they are inconsistent), there must be an entropy increase $\Delta S_{a}$ in the apparatus to complete the measurement. Hence, some energy $\Delta E_{1}=T_{a} \Delta S_{a}$, where $T_{a}$ is the temperature of the apparatus, must be transferred to the apparatus in the course of the measurement. (For simplicity, the possibility of cooling down some of the apparatus parts, which indeed can improve the detection, is not considered). Since $\Delta S_{a} \geq S_{3}$, and $S_{3} \sim k_{B}$ (we can assume $p_{\uparrow}=p_{\downarrow}=1 / 2$ for the estimates), the transferred energy must be sufficiently large $\Delta E_{1} \gtrsim k_{B} T_{a}$. Indeed, the measuring apparatus cannot detect any quantity that has energy effect comparable to $k_{B} T_{a} / 2$ - the average energy of thermal fluctuations associated with each excited degree of freedom. Detection needs a substantial initial energy effect $\Delta E_{1} \gg k_{B} T_{a} / 2$ that stands above the background of thermal fluctuations.

The initial effect needs to be amplified (say $\Delta E_{1} \longrightarrow \Delta E_{2} \gg \Delta E_{1}$ ) to reach macroscopic level and become easily detectable. A macroscopic object must stay reasonably close to a maximally mixed state since, otherwise, this process would spontaneously reduce entropy and violate the second law. As a thermodynamic object, the apparatus has a large number of degrees of freedom. While most of these degrees are microscopic, the final measurement state must be a macroscopic parameter or, otherwise, the results of measurement would remain undetected. Macroscopic parameters also fluctuate [47] but these fluctuations are very small $\sim k_{B} T_{a}$ compared to the average energy effect of the measurement $\sim \Delta E_{2}$. Any fluctuation or uncertainty that is much larger then $k_{B} T_{a}$ per degree of freedom is highly improbable according to the Gibbs distribution and, if created, would be promptly attenuated by the thermodynamic interactions. Once the measured value is amplified to macroscopic level, it becomes detectable with a high degree of certainty. The presence of an actual observer is not important - in this context "observable" means reaching macroscopic scales and producing a macroscopic effect which can be observed.

While decoherence is a very rapid process involving only very weak interactions with almost no energy transferred [44, 21, 23], the results of measurements can become known only after some time period, associated with energy transfer from the system to the apparatus (since, as discussed above, immediate availability of the results without energy transfer to the apparatus contradicts to the second law of thermodynamics). Therefore, information associated with the measurement is not available immediately after decoherence. Most acts of decoherence (and associated latent collapse) are not related to any measurements, never produce any macroscopic effect and are never observed. These acts do not involve observable collapses but, instead, increase uncertainty and entropy (i.e. $S_{0} \longrightarrow S_{3}$ ). Here, decoherences and latent collapses are understood as fundamental microscopic processes that underpin the macroscopic conduct of the second law. Since entropy increases on average everywhere in the universe, latent collapses must dominate observable collapses. This interpretation represents a thermodynamics-centred perspective on decoherence and collapse dictated by the Boltzmann time hypothesis.

\section{A.3 Everett's many-worlds interpretation}

It is useful to consider an alternative treatment of quantum measurements associated with Everett's many-worlds interpretation [48, 49]. This interpretation replaces collapse by the assumption of complete independence of the two states $|\uparrow\rangle$ and $|\downarrow\rangle$ after their decoherence. This independence persists forward in time creating two different "worlds" that remain independent of each other. Due to entanglement between the system, the apparatus, the observer, and, in fact, the rest of the universe, these worlds are slightly different having $|\uparrow\rangle\left|a_{\uparrow}\right\rangle\left|o_{\uparrow}\right\rangle$ in the world $\mathcal{W}_{\uparrow}$ and $|\downarrow\rangle\left|a_{\downarrow}\right\rangle\left|o_{\downarrow}\right\rangle$ in the world $\mathcal{W}_{\downarrow}$. As subjective observers, we can find ourselves only in one of these worlds $\mathcal{W}_{\uparrow}$ or $\mathcal{W}_{\downarrow}$ with the corresponding probabilities $p_{\uparrow}$ and $p_{\downarrow}$. While intellectually stimulating and original, the many-worlds interpretation is practically equivalent to collapse, as long as we do not know and cannot possibly predict a priori which of the worlds, $\mathcal{W}_{\uparrow}$ or $\mathcal{W}_{\downarrow}$, we will find ourselves in. Each of these branched worlds will split further and further with every irreversible act of quantum decoherence occurring anywhere in the universe.

Assigning a world split to every irreversible act of quantum decoherence corresponds the strong version of the Everett interpretation. Since a subjective observer finds himself only in one of the worlds, where only one of the states $|\uparrow\rangle$ or $|\downarrow\rangle$ is present, this decoherence thus appears in each of these worlds as a collapse. The key role of the strong version is thus in explaining the physical correspondence between decoherence and collapse. Since most of the collapses are latent, most of the splits produce 
macroscopically indistinguishable worlds. The subjective observer is located in one of the worlds but cannot know which one as many of the worlds appear to be identical under all possible observations. This excessive redundancy of the world splits can be removed by introducing a weak version of the Everett interpretation. This version proclaims that the world splits correspond only to observable collapses and, according to this version, all branched worlds are macroscopically different (the difference can be small or large but it must be macroscopic and observable).

Whether we might have many worlds or just one world in reality, the Everett interpretation is a very useful way of thinking about quantum mechanics replacing mysterious quantum randomness by branching the worlds, which, at least, is a useful way of thinking about randomness. This interpretation is attractive because it saves deterministic causality, which is deeply embedded into our intuition, from the harsh and unpredictable real world. Indeed, the future of the collective multiverse $\left\{\mathcal{W}_{\uparrow}, \mathcal{W}_{\downarrow}, \ldots\right\}$ is certain and fully determined by the past. It is only our subjective "I" that does not know which of these worlds it is going to find itself in. However, in the context of the present work, intuitive causality plays a negative role. Hence, we need to look at the Everett interpretation from a more time-symmetric perspective. The weak version of the interpretation is implied in the rest of the section.

Consider the initial state of the system and apparatus before the measurement

$$
\Psi_{0}^{\prime}=c_{\uparrow}|\uparrow\rangle\left|a_{0}\right\rangle-c_{\downarrow}|\downarrow\rangle\left|a_{0}\right\rangle
$$

Evolution of the system $\Psi_{0}^{\prime} \longrightarrow \Psi_{2}$ leads to the same outcome as considered previously $\Psi_{0} \longrightarrow \Psi_{2}$ : different initial conditions $\Psi_{0}$ in 100 and $\Psi_{0}^{\prime}$ in 25 result in, effectively, the same subsequent state $\Psi_{2}$ in (16) that corresponds to $\boldsymbol{\rho}_{2}=\boldsymbol{\rho}_{3}$. From the many-worlds perspective, this indicates a merger of two different worlds: the world $\mathcal{W}_{0}$ where $\Psi=\Psi_{0}$, and the world $\mathcal{W}_{0}^{\prime}$ where $\Psi=\Psi_{0}^{\prime}$ (assuming that the initial state of $\Psi$, has not been recorded anywhere or memorised by anyone despite being created in a lab, where the states $\Psi_{0}$ and $\Psi_{0}^{\prime}$ correspond to different positions of the macroscopic experimental controls). In the spirit of Everett's principles, any increase of entropy, which represents irreversible loss of information, involves merging of different worlds with different alternative pasts (in the same way as, splitting the worlds corresponds to different alternative futures). Hence, a consistent time-symmetric version of the many-worlds interpretation presumes existence of both the world mergers, e.g. $\left\{\mathcal{W}_{0}, \mathcal{W}_{0}^{\prime}\right\} \longrightarrow \mathcal{W}_{2}$ and the world splits, e.g. $\mathcal{W}_{2} \longrightarrow\left\{\mathcal{W}_{\uparrow}, \mathcal{W}_{\downarrow}\right\}$. The former is characterised by increases of the entropy $S_{0} \longrightarrow S_{2}$, the latter is associated with apparent decreases of the entropy $S_{2} \longrightarrow S_{4}$ (compensated by at least the same entropy increase in the apparatus or elsewhere). Since entropy tends to increase more than decrease locally and cannot decrease globally, we must expect that world merges dominate world splits. In other words, there are many possible alternative futures but even more possible alternative pasts (as discussed in section 5 and figure 3 , retrodicting is, typically, more difficult than predicting - this indicates that more alternatives exist in the past than in the future). Our perception that the past is fixed is created by our causality-based intuition; in fact, most of the past is irreversibly lost. Here, we refer to the whole body of information about the past, both unimportant and important (note that retrodiction can be easier than prediction when the sought information is selective - see examples 1-3 of section 44. Everett's interpretation has been long criticised for producing unphysically large numbers of multiplying and branching worlds, but in fact, variations in application of Everett's principles can easily produce more merges than splits. 


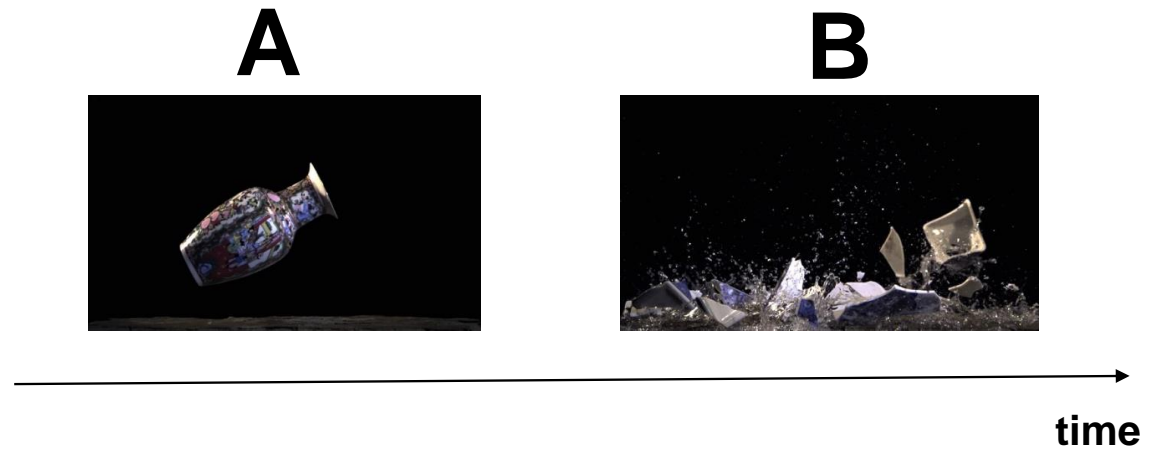

Figure 1: Casual and temporal precedence of two events.

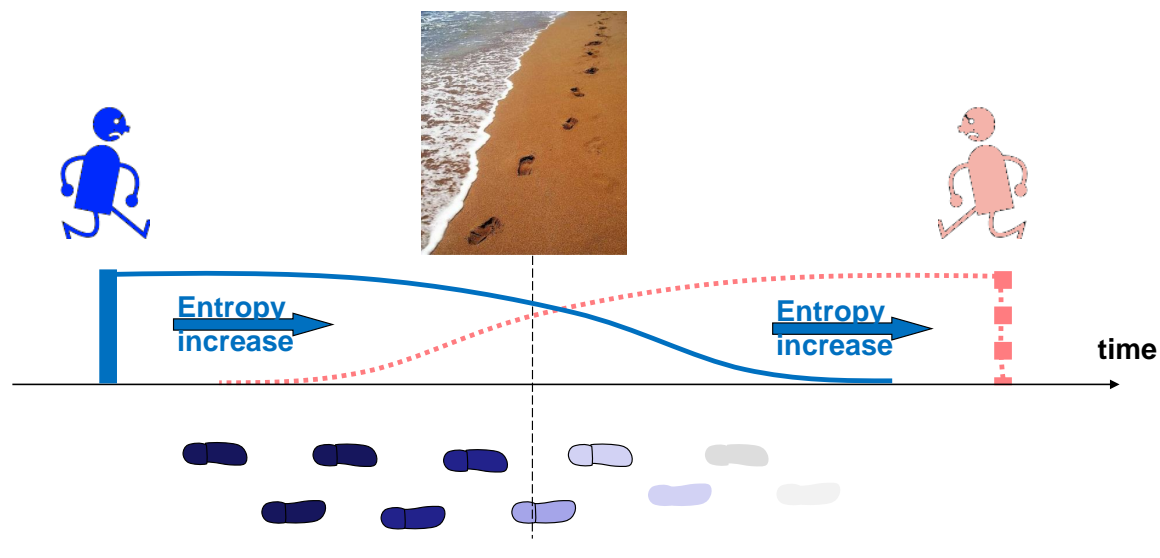

Figure 2: Causality, entropy increase and footsteps on a beach. The lines indicate the magnitude of the footprints: solid lines correspond to a realistic case, dashed lines violate the second law of thermodynamics and are not realistic. 


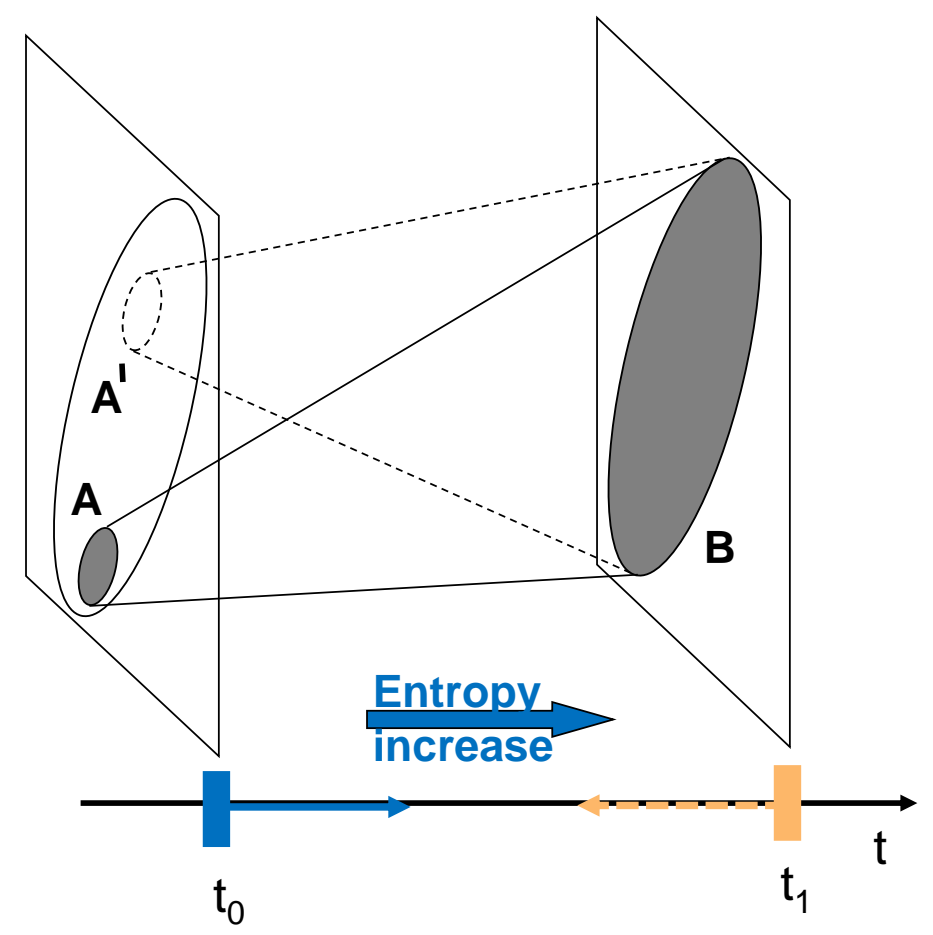

Figure 3: Practical causality - preference for initial (and not final) conditions - as an effect of the second law of thermodynamics. States A and $\mathrm{A}^{\prime}$ represent alternative initial conditions illustrated as different volumes in the phase space of a dynamic system, while state $\mathrm{B}$, which is close to equilibrium, is a common outcome of different evolutions of the system. The problem is well-posed forward in time and ill-posed backward in time.

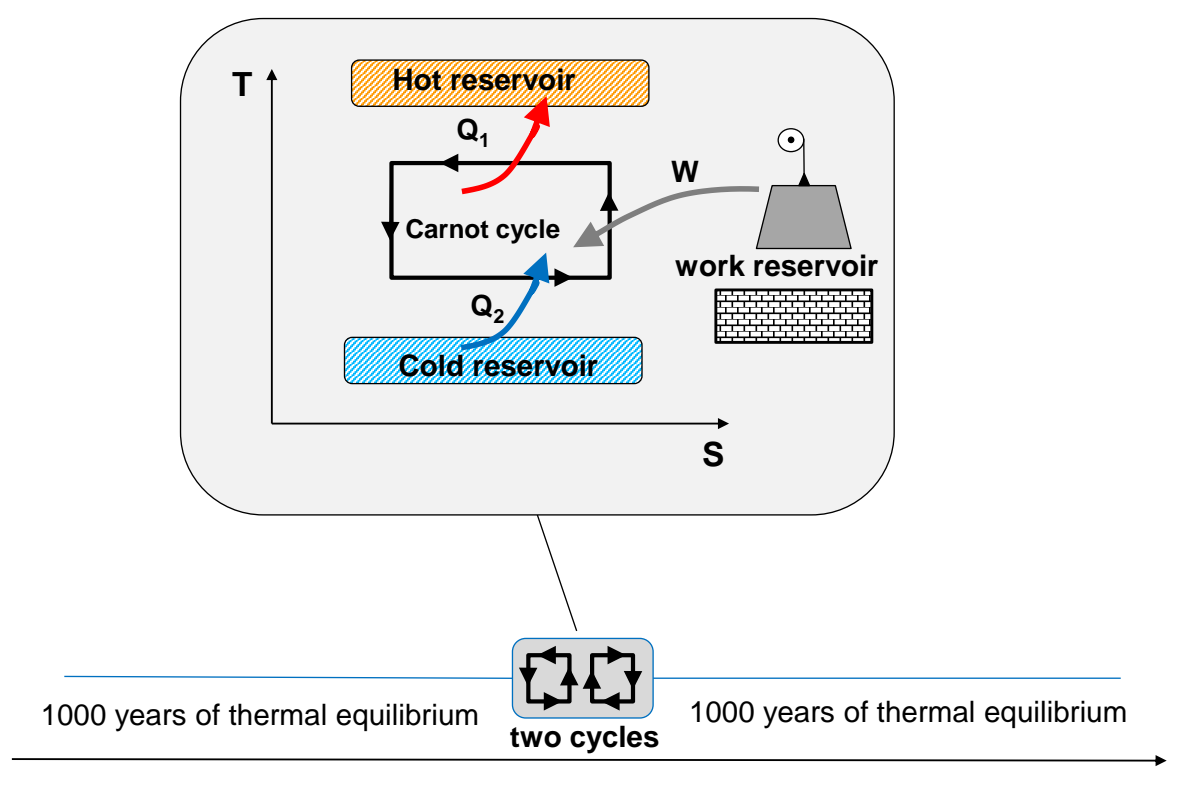

time

Figure 4: Using a Carnot engine to distinguish the directions of time in a remote part of the universe. The two cycles in the middle are screened from any influence of the rest of the universe by a remote location and thousand years of thermal equilibrium before and after activation of the cycles. 


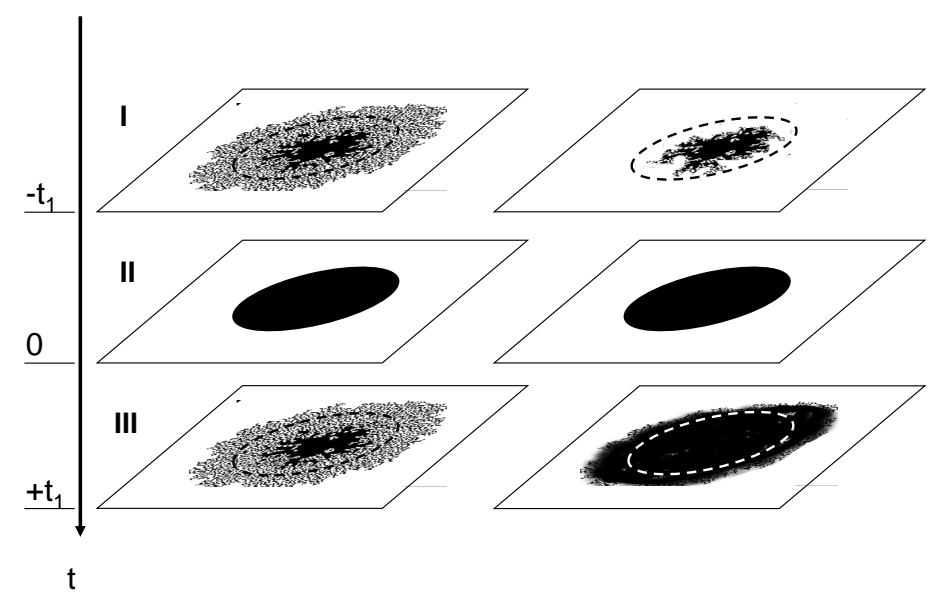

Figure 5: Illustration of ergodic mixing in a phase space of a dynamic system of a large dimension: a) without the time primer, the evolution is fully time symmetric and the black domain changes shape but has invariant phase volume associated with constant entropy; b) even tiny, almost undetectable, directional influence of the time primer induces macroscopic changes in the phase volume increasing the entropy forward in time.

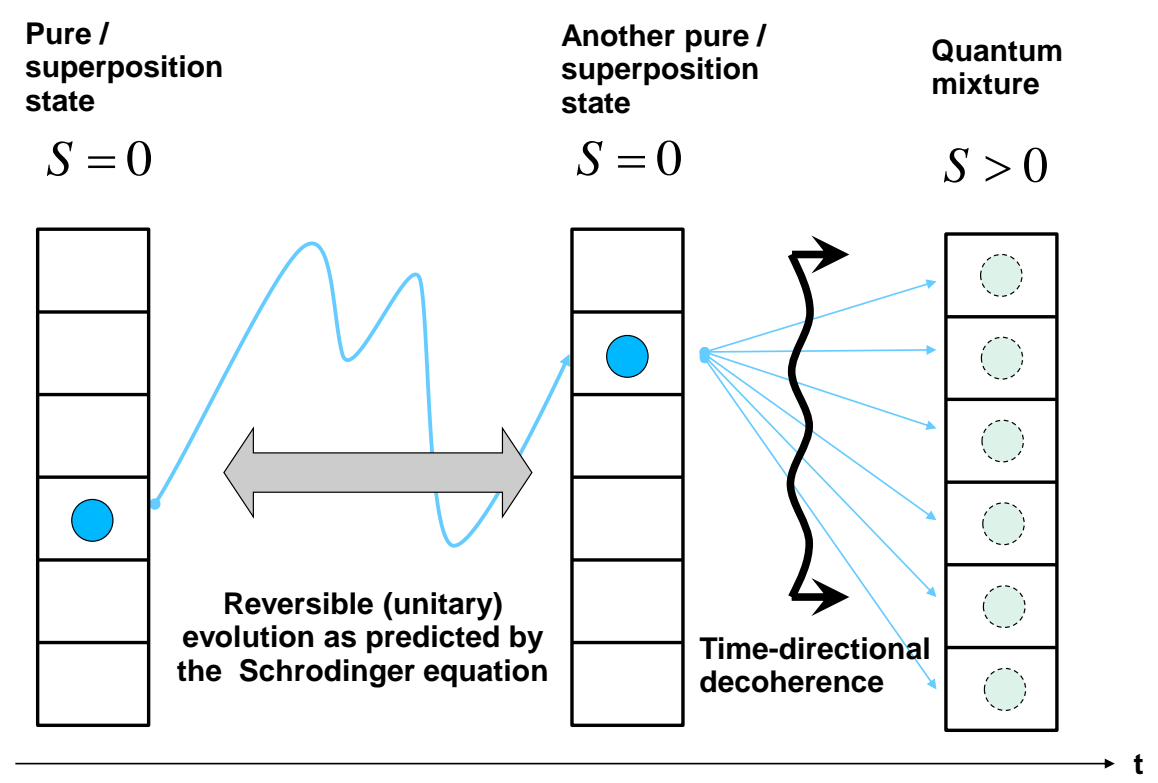

Figure 6: Quantum mixing and unitary evolution. Entropy remains constant in unitary evolutions but is increased during decoherence, which converts quantum pure states into quantum mixtures. 